\title{
Photochemistry of Reactive Intermediates
}

\author{
Thomas Bally*
}

\begin{abstract}
This paper illustrates the research on the photochemistry of reactive intermediates that was (and still is) carried out in the author's research group on three typical examples: the photochemistry of arylcarbenes, arylnitrenes, and organic radical cations.
\end{abstract}

Keywords: Carbenes $\cdot$ Nitrenes $\cdot$ Photochemistry $\cdot$ Radical ions $\cdot$ Reactive intermediates

\section{Introduction}

Reactive intermediates are often not present long enough and/or in sufficiently high concentration to be detectable during the course of thermal or photochemical reactions. In order to study the spectroscopic or kinetic properties of a reactive intermediate of interest, one must therefore be able to generate it by an independent, often a photochemical, pathway. Thus, carbenes and nitrenes can often be generated very efficiently by photolysis of their formal $\mathrm{N}_{2}$ adducts (diazirines, diazo compounds, azides), radicals and biradicals by photochemical extrusion of other suitable 'leaving groups' (iodine atoms, NO radicals, $\mathrm{CO}$, to name a few), and radical ions by photoinduced electron transfer. Thus, research on reactive intermediates is almost unthinkable without photochemistry. ${ }^{[1]}$

\footnotetext{
${ }^{*}$ Correspondence: Prof. Dr. T. Bally

University of Fribourg

Department of Chemistry

Chemin du Musée 9

$\mathrm{CH}-1700$ Fribourg

Tel.: +4126300 8705

Fax: +4126300 9737

E-Mail: Thomas.Bally@unifr.ch
}

However, the title of this account is not 'Reactive Intermediates by Photochemistry', but 'Photochemistry of Reactive Intermediates', which is quite a different subject. In fact, generating reactive intermediates under conditions where they persist, e.g. in low temperature frozen solutions or noble gas matrices, opens the possibility to probe not only their spectroscopic properties at leisure, but also their possible photochemical reactivity.

In previous contributions to topical CHIMIA issues, I have described other aspects of our program of research on reactive intermediates; ${ }^{[2,3]}$ here I will illustrate the title subject using examples taken from our work on three different kinds of reactive intermediates (carbenes, nitrenes, and radical cations)

\section{Technical Aspects}

As pointed out above, in order to study the photochemistry of reactive intermediates, these need to be stabilized, at least during the time it takes to effect a photochemical reaction and to probe the products. This is conveniently achieved by matrix isolation, nowadays a mature technique that is well documented. ${ }^{[4]}$ A specialty that we have developed in Fribourg is to generate radical ions in noble gas matrices (where molecules can be probed in transmission between 200 and 50 ' $000 \mathrm{~cm}^{-1}$ ) by exposing argon matrices containing the neutral precursors to X-irradiation. ${ }^{5]}$ It is important to realize that organic molecules are practically transparent to X-rays, whereas Ar has a large cross section for their absorption and scattering, processes which result in the creation of electron-hole pairs. A small fraction of these pairs escape recombination and travel through the Ar matrix (presumably in the form of polarons), until the holes encounter species that have a lower oxidation potential than $\mathrm{Ar}$ (which is the case for most organic substrates) and the electrons are scavenged by a species that has a higher electron affinity than $\mathrm{Ar}$ (which, for example, most alkyl halides do). Thus, radical ions are not formed under the direct impact of X-rays (which, if absorbed, would probably knock any polyatomic molecule to pieces), but by charge transfer from $\mathrm{Ar}^{\circ+}$ or $\mathrm{Ar}^{-}{ }^{-}$. After thermalization, radical ions are for all practical purposes indefinitely stable at $10 \mathrm{~K}$, the temperature at which Ar matrices are usually held.

A second technical aspect concerns the photoreactions: normally, such processes are effected simply by exposing samples to the light of some commercial light source such as a $\mathrm{Xe} / \mathrm{Hg}$ arc, perhaps passing the light through a UV cutoff filter. However, reactive intermediates, in particular radical ions, often absorb throughout the visible and sometimes even the NIR range, so indiscriminate irradiation often leads to a mixture of primary and secondary products which is then hard to disentangle. Therefore, whenever we study the photochemistry of reactive intermediates, we start by irradiating at the longest wavelength where we can discern an absorption. When this does not lead to the disappearance of that absorption, we carefully inch towards shorter wavelengths, using an array of interference and cutoff filters, until the system under investigation shows a change. Sometimes we even have to go as far as to use a monochromator to give us light of a desired wavelength (of course this only works if a photoconversion occurs with a high quantum yield). This strategy has allowed us to sort out rather complicated schemes of sequential and parallel photochemical reactions to which reactive intermediates may be subjected. 


\section{Photochemistry of Arylcarbenes}

Arylcarbenes usually have triplet (T) ground states (but very low-lying singlet states that may even be in thermal equilibrium with the triplet at room temperature). In low-temperature matrices the bimolecular processes of the incipient singlet (S) carbenes that are responsible for their "chemical usefulness', as well as possible unimolecular decay processes, such as rearrangement to cycloheptatetraenes (see Scheme 1), are suppressed. Therefore, intersystem crossing (ISC) will dominate, and one can only observe triplet arylcarbenes in matrices. ${ }^{6}$ We proposed that, after threshold photoexcitation, triplet phenylcarbene (1) might remain on the triplet surface long enough to undergo rearrangement to the elusive triplet cycloheptatrienylidene carbene ${ }^{32}$. However, it seems that at some point of this reaction, the system undergoes efficient ISC, because the only product we could observe from this photoreaction was the previously known nonplanar cycloheptatetraene (2). Nevertheless, this experiment allowed us to fully characterize the electronic structure and the force field of the parent triplet phenylcarbene (1) and cycloheptatraene (2). ${ }^{7]}$

In another of our encounters with carbene chemistry, we were confronted with an enigmatic photorearrangement of triplet 2-naphthyl(carbomethoxy)carbene ${ }^{3} \mathbf{3}$ leading to a species with a broad absorption at $420 \mathrm{~nm}$. We could indeed observe this spectral change in Ar matrices, but in addition, Zhendong Zhu found that the reaction could be reverted, both by irradiation at 450 $\mathrm{nm}$ and, more surprisingly, by letting a matrix stand overnight at $12 \mathrm{~K}$. Although, by virtue of this reversibility, we had full UV/ Vis and IR spectra of the rearrangement product, this species resisted our attempts to provide a plausible assignment until $\mathrm{Zhu}$ came up with the somewhat iconoclastic proposition that this product might be the singlet state of $\mathbf{3}$, a proposition that initially met with much skepticism on the part of the 'carbene community'. In spite of that we eventually succeeded, with the help of our IR spectra (Fig. 1B) and the ESR spectra in Ar matrices that were kindly provided by Prof. McMahon, to assemble sufficient convincing evidence that this assignment was accepted as the first example of 'spin isomerism', i.e. the interconversion between two (meta)stable spin states of the same species (Scheme 2). ${ }^{[8]}$

It appears that it was a fortunate combination of circumstances that allowed us to make this discovery: Not only are the two spin states very close in energy, ${ }^{[9]}$ but on $\mathrm{T} / \mathrm{S}$ interconversion the COOMe group assumes an orthogonal conformation which provides for a small barrier for the decay of the less stable singlet state to the triplet ground state. ${ }^{[8]}$

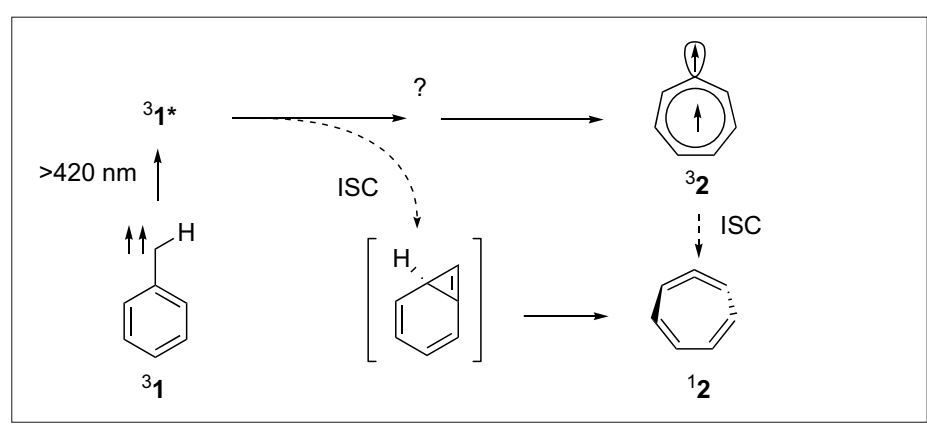

Scheme 1.

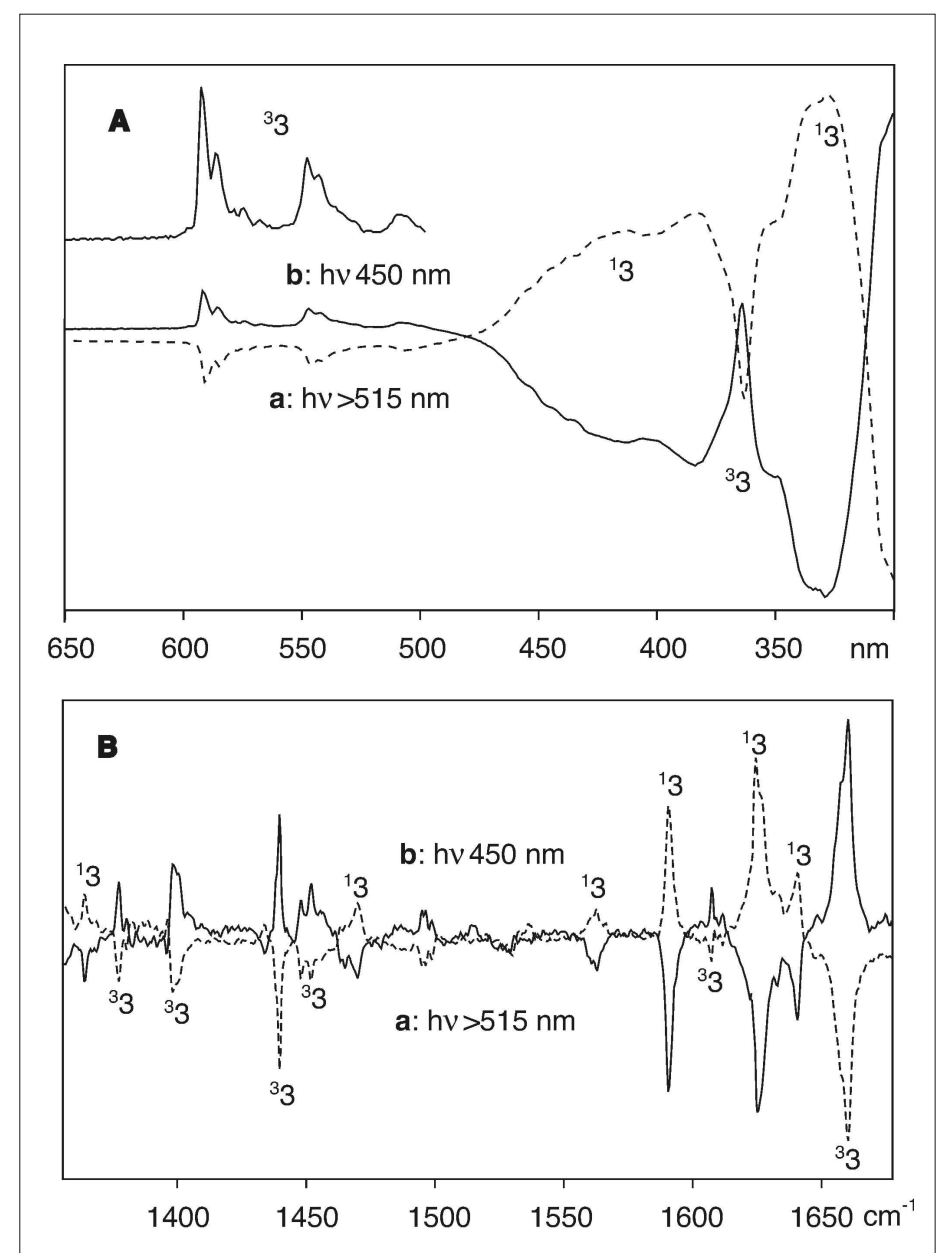

Fig 1. UV-Vis (A) and IR (B) spectral changes for the reversible interconversion of the singlet and triplet states of 2-(carbomethoxy)naphthyl nitrene 3 .

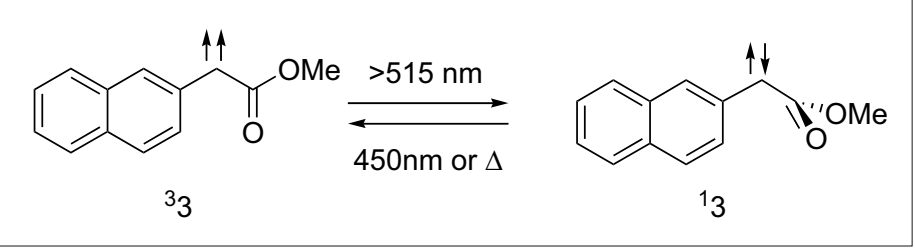

Scheme 2.

\section{Photochemistry of Arlynitrenes}

Nitrenes are iso- $\pi$-electronic to the corresponding carbenes (one $\mathrm{C}-\mathrm{H}$ bond being formally replaced by a lone pair on the nitrogen atom). Arylnitrenes also have triplet ground states, but the singlet-triplet gap is usually much bigger $(\mathrm{ca} .18 \mathrm{kcal} / \mathrm{mol}$ in phenylnitrene), and intersystem crossing is slower than in comparable arylcarbenes.[10] On the other hand the barriers for expansion of arylnitrenes to azacycloheptatetraenes (or dehydroazepines) are lower than those for the corresponding arylcarbene rearrangements. [11] As dehydroazepines polymerize readily, arylnitrenes were long regarded as 
chemically useless, until it was discovered that ortho-bisubstituted arylnitrenes resist rearrangement to an extent that bimolecular reactions of singlet arylnitrenes can be exploited, for example in photoaffinity labelling ${ }^{[12]}$ or in imaging applications. ${ }^{[13]}$

$o$-Bisubstitution not only raises the barrier for the attack of the nitrene's $\mathrm{N}$-atom on the aryl ring, but also that for the subsequent opening of the bicyclic compound to form the dehydroazepine. [11] This opens the possibility to observe all three species, and we have succeeded in the case of 2,5difluorophenylnitrene (4). We found that the three reactive intermediates which are in equilibrium at room temperature, i.e. the nitrene 4 , azirine 5 , and dehydroazepine 6 (Scheme 3), can be interconverted by selective photolyses and characterized by their full UV/Vis and IR spectra. ${ }^{[14]}$

If naphthylnitrenes undergo the above type of rearrangement, this may entail the loss of an aromatic ring which would be expected to affect the thermochemistry. We thus set out to explore the photochemistry of naphtylnitrenes and thereby made an interesting discovery: instead of forming, from the appropriate azirines which can easily be generated, the high-energy $o$-quinoid dehydroazepines (which, if formed, would in any event decay back to the percursor azirines in practically barrierless processes, as predicted by calculation), these systems undergo a bond-shift isomerization to form novel ylidic compounds, as exemplified below for the case of 1-naphthylnitrene (7).

The UV/Vis and IR-spectra obtained on $313 \mathrm{~nm}$ bleaching of 1-naphthylazide are too complex to be analyzed in terms of a single product. Careful photolyses allow, however, to selectively affect different constituents of this mixture and to identify eventually the triplet nitrene, ${ }^{3} 7$, the azirine, $\mathbf{9}$, and the aromatic dehydroazepine $\mathbf{8}$ (Scheme 4). However, the spectra of the last of the expected constituents of the mixture, the $o$-quinoid dehydroazepine 11, could not be brought into agreement with those calculated by quantum chemical methods. This forced us to consider alternative hypotheses for this species, the spectra of which we had in our hands.

It then happened that students in three research groups (Alexander Maltsev in our group, Meng-Lin Tsao in the group of Prof. Platz at Ohio State University and Arvid Kuhn in the group of Prof. Wentrup at the University of Queensland in Australia) independently discovered that the missing dehydroazepine $\mathbf{1 1}$ existed in the form of a more stable ylidic structure $\mathbf{1 0}$ which restores the aromaticity in the benzene ring. The existence of such species had been postulated earlier by calculations, but the careful photochemical work of Alexander

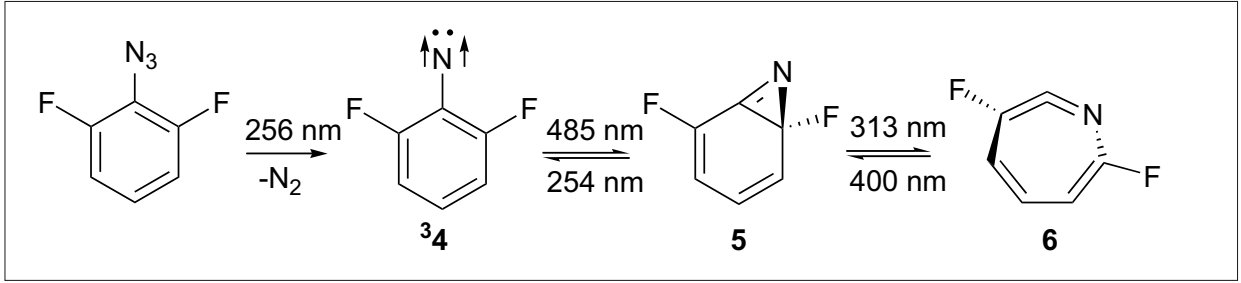

Scheme 3.

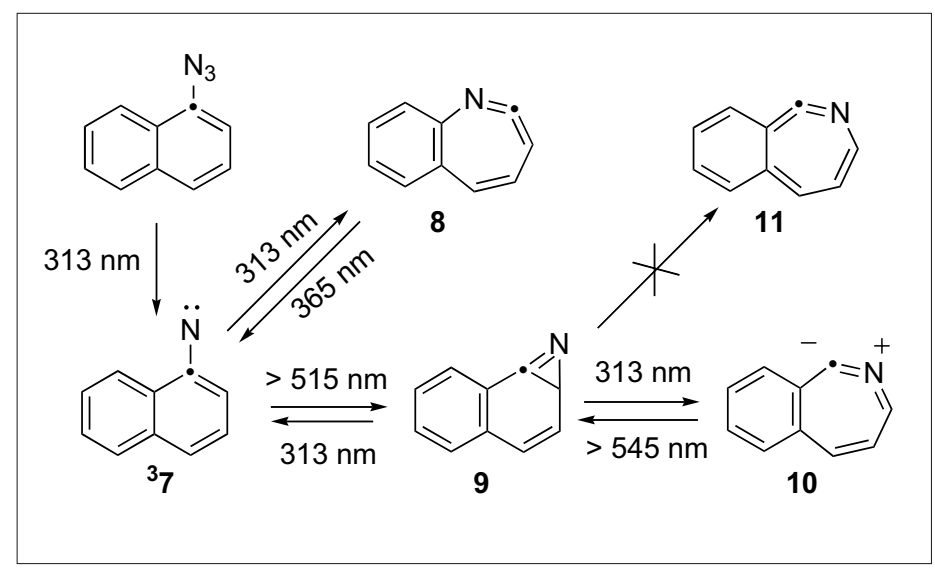

Scheme 4

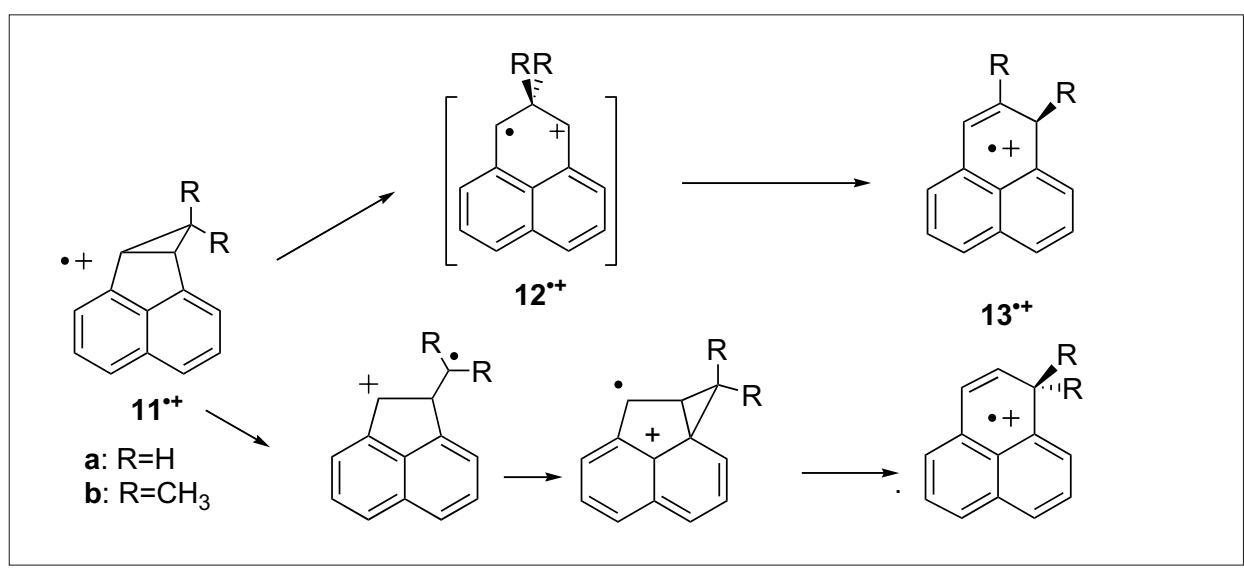

Scheme 5.

Maltsev allowed these novel species to be isolated and unambiguously characterized for the first time. Eventually the three groups teamed up to publish these findings. [15]

We have also recently explored the 'internal trapping' of phenylnitrenes by nucleophiles or by biradicaloid pathways, a study that involved a lot of photochemistry. [16]

\section{Photochemistry of Radical Cations}

Radical cations often show rich electronic absorption spectra that extend throughout the visible, and sometimes even into the near IR-range, which offers the opportunity to induce photochemical reactions by selective irradiations. In this way we have studied several photoinduced ring-open- ing reactions of radical cations containing three-[17-21] or four-membered rings, ${ }^{[22-25]}$ phototautomerizations of ionized ketones and enols, ${ }^{[26-28]}$ and rotamer interconversions in polyene radical cations. ${ }^{[29-31]}$ I will use two examples to illustrate the photochemistry of radical cations:

Photolysis of the radical cations of perinaphthocyclopropanes $\mathbf{1 1}^{\mathbf{*}}$ leads directly to phenalene radical cations $\mathbf{1 3}^{\mathbf{*}}($ Scheme 5$)$, irrespective of whether $\mathrm{R}=\mathrm{H}$ or $\mathrm{CH}_{3}$, i.e. the primary 1,3-perinaphthadiyl radical cations $\mathbf{1 2}{ }^{*}$, if they are formed at all, decay spontaneously. (In fact, calculations indicate that, in the case of $\mathrm{R}=\mathrm{CH}_{3}$, the phenalene radical cation is formed by initial lateral cleavage of the cyclopropane ring, followed by rearrangement to $\mathbf{1 3}^{\circ+}$. Unfortunately we were not able to distinguish the two isomeric forms of 13b+ by optical spectroscopy). 
However, if formed from the doubly bridged compound $\mathbf{1 4}$ the corresponding 1,3-perinaphthadiyl radical cation $\mathbf{1 5}^{\mathbf{}}+$ is stable, and it can in turn be converted to the radical cation of the cross-conjugated polyene, 2,7-dihydro-2,2,7,7-tetramethylpyrene, $\mathbf{1 6}^{\mathbf{0}}$ (which was generated independently from neutral 16) (Fig. 2). ${ }^{[18]}$

This example demonstrates the importance of selective photolysis: on irradiation of $\mathbf{1 4}^{-+}$through a simple UV-cutoff filter, the spectrum of $15^{\circ+}$ never appears, because ring-opening to $\mathbf{1 6}^{\mathbf{}}$ occurs with a much higher quantum yield than formation from $14^{+} .15^{\circ+}$ can only be formed and observed under conditions of irradiation where it does not absorb.

The radical cation of perinaphthocyclobutane $\mathbf{1 7}^{\circ+}$ (Scheme 6) also proved to be photolabile and undergoes ring opening to a 1,4-perinaphtadiyl radical cation $\mathbf{1 8}^{\circ+}$ (which we could generate independently from the corresponding azo precursors). On higher energy photolysis, cleavage of the second $\sigma$-bond occurred and the radical cation of 1,8-divinylnaphthalene $\mathbf{1 9}^{-+}$was formed. Again, intermediate $\mathbf{1 8}^{\mathbf{+}}$ cannot be observed under full visible irradiation. ${ }^{[22]}$

The last example comes from our work on electron-transfer-induced tautomerizations, ${ }^{[26]}$ and this example taught us to watch out for subtle electronic factors in organic radical cations.

On ionization, dimethylindanone $\mathbf{2 0}$ spontaneously undergoes partial tautomerization to the more stable $o$-quinoid enol $21^{-+}$ (Fig. 3, Scheme 7) which distinguishes itself by a well-resolved absorption band around $700 \mathrm{~nm}$. On irradiation of this band $(\lambda>640$ $\mathrm{nm}), \mathbf{2 1}^{\circ+}$ is bleached and $\mathbf{2 0}{ }^{\circ+}$ is reformed, as shown very clearly by the IR spectra. On irradiation at $490 \mathrm{~nm}$, or on standing overnight at $12 \mathrm{~K}$, the spectrum of $\mathbf{2 1}^{\mathbf{}}+$ can be fully recovered. Replacing the two methyl groups in 20 by $\mathrm{CD}_{3}$-groups has no influence on the photochemical behavior of the system, but the spectrum of the ketone radical cation is indefinitely stable at $12 \mathrm{~K}$ (or even at $25 \mathrm{~K}$ ), which suggests that tunnelling is involved in the tautomerization.

To our surprise we found that, on ionization of methylindanone 22, there was no trace of the keto radical cation, $\mathbf{2 2}^{*}$, in the spectra, and that the corresponding enol radical cation, $\mathbf{2 3}^{\mathbf{}}$, was entirely photostable. However, deuteration of the methyl group in $\mathbf{2 2}$ fully restored the phototautomerism observed in $\mathbf{2 0}^{\mathbf{*}} / \mathbf{2 1}^{\circ+}$. Apparently the rate of tunnelling is several orders of magnitude higher in $\mathbf{2 2}^{\mathbf{*}}$ than it is in $\mathbf{2 0}^{\circ+}$ so that the former immediately decays to $\mathbf{2 3}^{*+}$ (possibly $\mathbf{2 2}^{*+}$ could be generated by flash photolysis and observed by time-resolved spectroscopy, but this cannot be readily done in matrix isolated samples).[28]

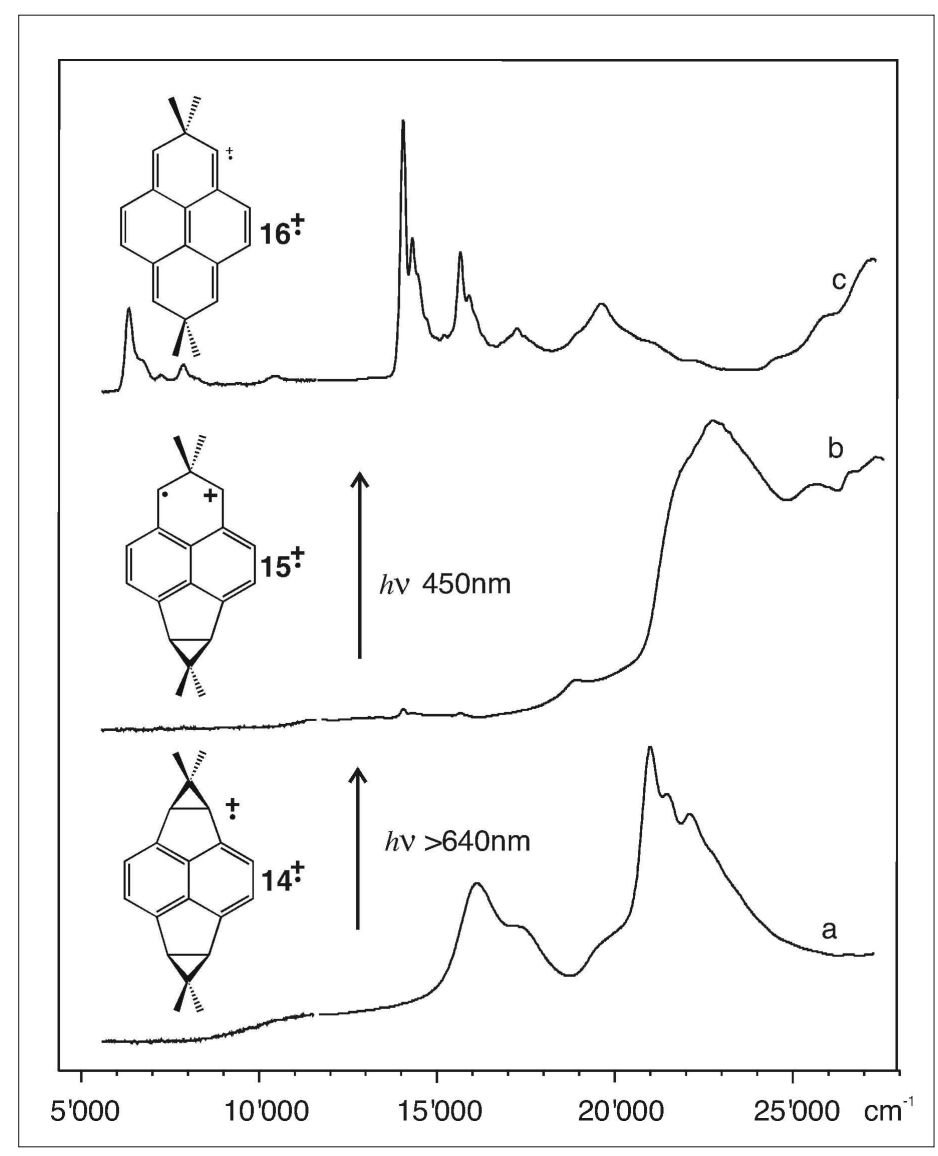

Fig 2. Changes in the electronic absorption spectra on generation of the radical cation of naphthalene derivative 14 and its subsequent conversion to diradical cation $16^{\circ+}$ and the radical cation of the cross-conjugated dihydropyrene 17

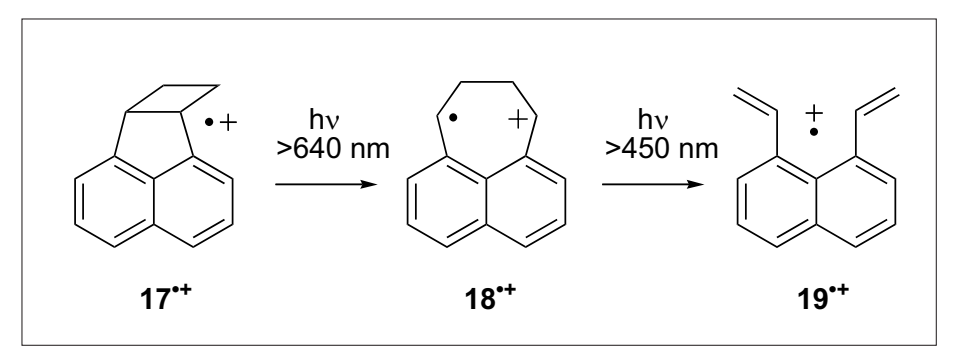

Scheme 6.

These results raised the question of why and how a remote methyl group can so strongly influence the tautomerization kinetics of the radical cation of methylindanone. Indeed calculations showed that the additional methyl group in $\mathbf{2 0}^{-+}$raises the barrier for enolization by $c a .6 .5 \mathrm{kcal} / \mathrm{mol}$, enough to explain our observations.

The reason for this lies in the electronic structure of aromatic ketone radical cations: Enolization can only occur from a state where an electron is missing from an oxygen lone pair (which is then poised to abstract a hydrogen atom from a vicinal alkyl group), whereas states where the electron is missing from the $\pi$-system are unreactive with regard to $\mathrm{H}$-abstraction. In $\mathbf{2 2}^{\mathbf{*}}$ these two states are nearly degenerate, whereas in $\mathbf{2 0}^{*+}$ the ground state arises from ionization of a $\pi$-MO while the $\sigma$-radical state lies 6.5 $\mathrm{kcal} / \mathrm{mol}$ higher in energy. Once this reactive state has been reached, the enolization kinetics of $\mathbf{2 0}^{\mathbf{*}}$ and $\mathbf{2 2}^{\mathbf{}}+$ are nearly identical. Thus, the difference in reactivity of these two very similar compounds are a result of the stabilization of the unreactive $\pi$-radical state by the additional methyl group. ${ }^{[28]}$

This example shows that it is worthwhile to pay close attention to proximate electronic states in radical cations, because the photochemical and thermal reactivity of radical cations can be very different in different electronic states.

\section{Conclusion}

This account gives a flavor of the interesting photochemistry that different reactive intermediates reveal under conditions 

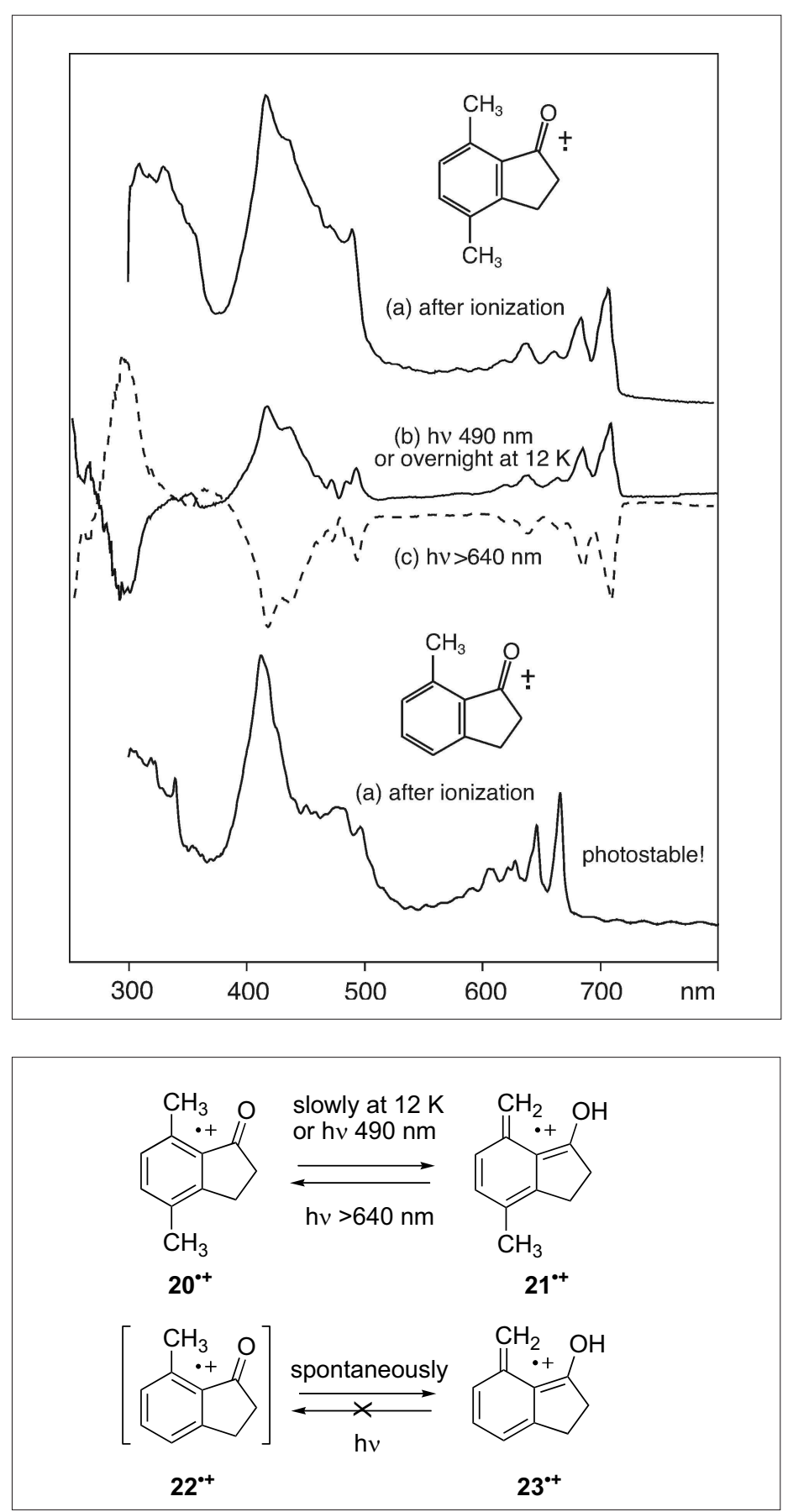

where they are sufficiently stable to undergo light-induced reactions, and that this photochemistry is worthwhile exploring, not only for its academic interest, but also in view of the fate of reactive intermediates in various applications.

\section{Acknowledgments}

The work described above would of course have been impossible without the hard work of the very dedicated graduate students and postdocs who have contributed to it. In the present context I owe particular gratitude to Stephan Matzinger, Zhendong Zhu, Alexander Maltsev, Beat Müller, Claudio Carra and Juliane Grota. I would also like to thank Prof. Edwin Haselbach who initiated me over 20 years ago to the photochemistry of radical cations. [32] Finally, I would like to express my thanks to the Swiss National Science Foundation whose
Fig. 3. (a) Changes in the UV/Vis spectra on ionization of methyl- or dimethylindanone $(22$ or $\mathbf{2 0}$, respectively) which results in its partial conversion to the corresponding enol radical cations (see Scheme 7); (b) and (c) reversible interconversion of $\mathbf{2 0}^{\circ+}$ and $21^{\circ+}$ by selective photolysis or, in the case of the reaction leading to the enol radical cation, thermally at $12 \mathrm{~K}$ (see text).

Scheme 7.

continued support was of course essential for the achievements described in this brief account.

Received: July 25, 2007

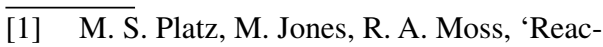
tive Intermediate Chemistry', John Wiley \& Sons, Hoboken, NJ, 2004.

[2] T. Bally, Chimia 1994, 48, 378.

[3] T. Bally, Chimia 2005, 59, 522.

[4] See, e.g. T. Bally, in ref. [1], p. $820 \mathrm{ff}$. and references cited therein.

[5] T. Bally, in 'Radical Ionic Systems', Vol. 6, Eds. A. Lund, M. Shiotani, Kluwer, Dordrecht, 1991, pp. 3.

[6] See, e.g. S. Matzinger, T. Bally, E. V. Patterson, R. J. McMahon, J. Am. Chem. Soc. 1996, $118,1535$.
[7] S. Matzinger, T. Bally, J. Phys. Chem. A 2000, 104, 3544.

[8] Z. Zhu, T. Bally, L. L. Stracener, R. J. McMahon, J. Am. Chem. Soc. 1999, 121, 2863.

[9] Y. Wang, C. M. Hadad, J. P. Toscano, J. Am. Chem. Soc. 2002, 124, 1761.

[10] W. T. Borden, N. P. Gritsan, C. M. Hadad, W. L. Karney, R. K. C, M. S. Platz, Acc. Chem. Res. 2000, 33, 765.

[11] W. L. Karney, W. T. Borden, J. Am. Chem. Soc. 1997, 119, 1378.

[12] H. Bayley, 'Photogenerated Reagents in Biochemistry and Molecular Biology', Elsevier, Amsterdam, 1983.

[13] N. J. Clecak, R. J. Cox, W. M. Moreau, Polym. Eng. Sci. 2004, 14, 491.

[14] C. Carra, R. Nussbaum, T. Bally, ChemPhysChem 2006, 7, 1268.

[15] A. Maltsev, T. Bally, M.-L. Tsao, M. S. Platz, K. A., M. Vosswinkel, W. C., J. Am. Chem. Soc. 2004, 126, 237.

[16] C. Carra, T. Bally, A. Albini, J. Am. Chem. Soc. 2005, 127, 5552.

[17] T. Bally, S. Nitsche, E. Haselbach, Helv. Chim. Acta 1984, 67, 86.

[18] T. Bally, Z. Zhu, J. Wirz, M. Fülscher, J.-Y. Hasegawa, J. Chem. Soc. Perkin Trans. 2 2000, 2311.

[19] B. Müller, T. Bally, F. Gerson, A. de Meijere, M. von Seebach, J. Am. Chem. Soc. 2003, 125, 13776.

[20] C. Gaebert, J. Mattay, M. Toubartz, S. Steenken, B. Müller, T. Bally, Chem. Eur. J. 2005, 11, 1294.

[21] T. Bally, B. Müller, F. Gerson, X.-Z. Qin, M. von Seebach, S. I. Kozhushkov, A. de Meijere, V. I. Borovkov, P. A. Potashov, J. Phys. Chem. A 2006, 110, 1163.

[22] Z. Zhu, T. Bally, J. Wirz, M. Fülscher, J. Chem. Soc. Perkin Trans. 2 1998, 1083.

[23] T. Bally, S. Bernhard, S. Matzinger, J.-L. Roulin, G. N. Sastry, L. Truttmann, Z. Zhu, A. Marcinek, J. Adamus, R. Kaminski, J. Gebicki, F. Williams, G.-F. Chen, M. P. Fülscher, Chem. Eur. J. 2000, 6, 858.

[24] K. Schroeter, D. Schröder, H. Schwarz, G. D. Reddy, O. Wiest, C. Carra, T. Bally, Chem. Eur. J. 2000, 6, 4422.

[25] J. Grota, J. Mattay, K. Piech, T. Bally, Chem. Eur. J. 2006, 12, 4559.

[26] J. Gebicki, T. Bally, Acc. Chem. Res. 1997, 30, 477.

[27] A. Marcinek, J. Adamus, K. Huben, J. Gebicki, T. J. Bartczak, P. Bednarek, T. Bally, J. Am. Chem. Soc. 2000, 122, 437.

[28] P. Bednarek, Z. Zhu, T. Bally, T. Filipiak, A. Marcinek, J. Gebicki, J. Am. Chem. Soc. 2001, 123, 2377.

[29] T. Bally, S. Nitsche, K. Roth, E. Haselbach, J. Phys. Chem. 1985, 89, 2528.

[30] T. Bally, S. Nitsche, K. Roth, J. Chem. Phys. 1986, 84, 2577.

[31] T. Bally, E. Haselbach, S. Nitsche, K. Roth, Tetrahedron 1986, 42, 6325.

[32] E. Haselbach, T. Bally, Pure Appl. Chem. 1984, 56, 1203. 